\title{
Recent Developments in the ATLAS Pixel Detector
}

\author{
Francesco Ragusa $^{\mathrm{a}, 1}$

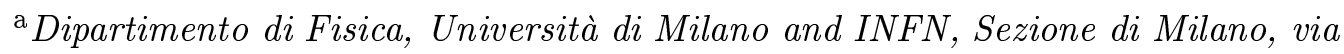 \\ Celoria 16, I-20133 Milano, Italy
}

\begin{abstract}
Silicon pixel detectors, developed to meet LHC requirements were tested in 1998 and 1999 within the Atlas collaboration in the H8 beam test at CERN. Different sensor designs have been developed and studied using an analog front end electronics chip. In this work a detailed experimental study of the overall performance of both irradiated and not irradiated detectors for the different designs is presented, with special emphasis to efficiency, charge collection and resolution measurements. Beahvior of detectors in a magnetic field is also discussed.
\end{abstract}

\section{Introduction}

The ATLAS Pixel Detector [1] will be operated in a severe environment where a high efficiency has to be achieved despite the radiation damage and the challenging high data acquisition rate. To meet these requirements a special effort is in progress to develop radiation resistant sensors and front end electronics. Details of electronics and sensors designs are given elsewhere $[1,2]$; here we recall some basic aspects important to discuss the results presented here.

Front end electronics. To achieve high data acquisition rate with the high luminosity LHC machine the

1 On Behalf of the ATLAS Pixel Collaboration[1]

Preprint submitted to Elsevier Preprint data of a pixel hit (address, pulse amplitude, bunch crossing number) selected by a Level 1 Trigger will be stored in the front end chip, operated synchronously with the $40 \mathrm{MHz}$ machine R.F. Subsequently, if the event is selected by higher level triggers, the hit will be read-out from the front end chip local buffers using the stored bunch crossing number. The pulse height measurement is performed by measuring the time the pulse from the amplifier remains above the threshold (Time Over Threshold). During the operation in the test beam the thresholds of the individual channels were adjusted achieving a threshold dispersion of 170 electrons rms. Typical thresholds were around 3000 electrons, going down to 2000 electrons for charge collection studies. The noise 


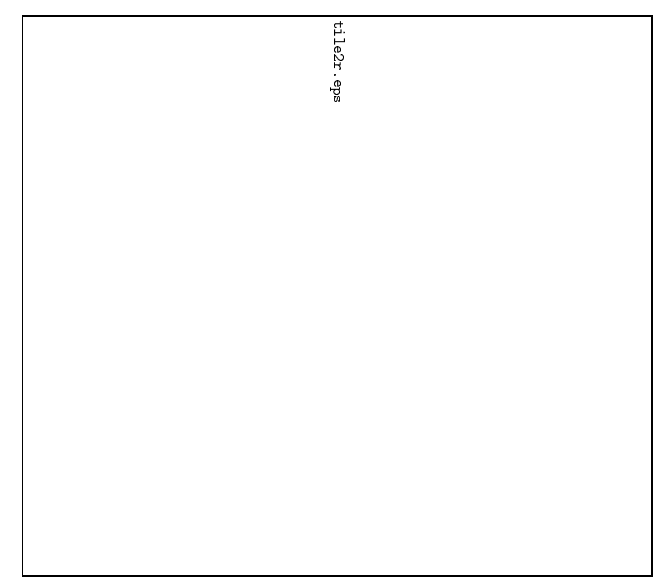

Fig. 1. The first Tile 2 sensor design.

was typically 150 electrons rms. The TOT was calibrated by injecting a known charge to every channel, with an accuracy of the order of $5 \%$.

Pixel detectors. A module of the ATLAS pixel detector consists of a Tile containing an array of $2 \times 8$ sensors. On each sensor, a front end electronic chip is bump bonded using Indium bumps or $\mathrm{SnPb}$ solders. The results reported in this paper are based on data collected with single chip assemblies made of one single sensor with a front end chip bump bonded on it. The pixel cells, $400 \mu \mathrm{m}$ times $50 \mu \mathrm{m}$ in size, were obtained by $n^{+}$implants on $n$-type substrate; to achieve the pixels electrical insulation two different solutions were used producing two sensor designs: the so called Tile 1 design in which the pixel cells were insulated using high dose $p$-stop implants [2,3] surrounding the pixel cells and the Tile 2 design in which the insulation was obtained by employing the p-spray technique $[2,4]$. The Tile 2 design also features a bias grid, used to bias all the pixels by the punch through mechanism to test the sensors before

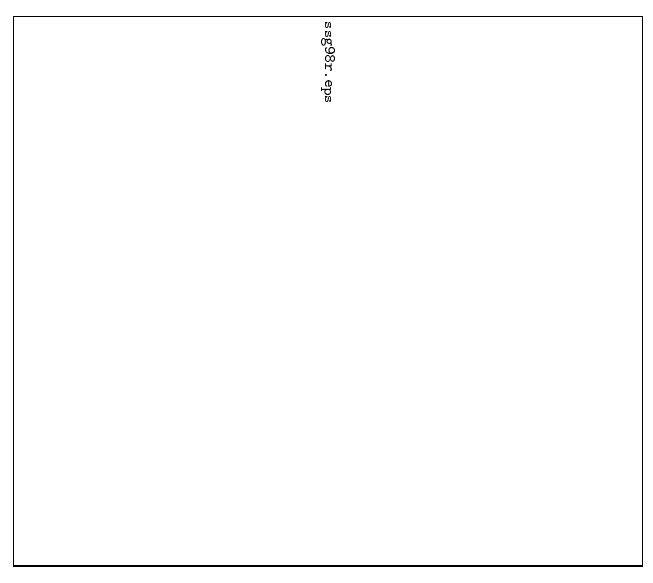

Fig. 2. The new Tile 2 sensor design.

bump bonding to front end chips; during normal operation the grid is not used and the bias to the pixels is given by the connected preamplifiers. As will be shown in sections 2 and 3 the first Tile 2 design (Fig. 1) had a charge collection problem related to the presence of the floating atoll surrounding the pixel whose purpose was to reduce the interpixel capacitance; a new design still employing the p-spray insulation technique in which the floating atoll was removed (Fig. 2) was developed and studied in the test beam. To asses the radiation resistance of the sensor, some detectors were exposed to a flux comparable to those expected for LHC. Irradiations were performed using the $300 \mathrm{MeV} / \mathrm{c}$ pion beam at PSI and the $55 \mathrm{MeV} / c$ proton beam at LBNL. In what follows the doses will be converted in $\mathrm{n}_{\mathrm{eq}} / \mathrm{cm}^{2}$ where $\mathrm{n}_{\text {eq }}$ is the number of particles with the non-ionising energy loss of a 1 $\mathrm{MeV}$ neutron [5,6]. Sensors irradiated with fluences of $0.5 \times 10^{15}$ and $1 \times 10^{15} \mathrm{n}_{\mathrm{eq}} / \mathrm{cm}^{2}$ were tested, the last one corresponding approximately to 5 years of LHC operation for the first barrel layer and 10 years for the sec- 
ond barrel layer at $4.3 \mathrm{~cm}$ and 10.1 $\mathrm{cm}$ from the beam line respectively; the lifetimes calculations assume a linear increase of the luminosity for the first 3 years from $10^{33} \mathrm{~cm}^{-2} \mathrm{~s}^{-1}$ to the nominal design luminosity of $10^{34}$ $\mathrm{cm}^{-2} \mathrm{~s}^{-1}$. The irradiated sensor were kept at $-10^{0} \mathrm{C}$ during data taking.

Test beam setup. The studies presented in this paper were done at the CERN SPS accelerator with a pion beam of $180 \mathrm{GeV} / c$ momentum. During the extraction, the beam lost his R.F. structure and the particles crossing the detector had a random phase with respect to the front end electronics $40 \mathrm{MHz}$ clock: this phase was measured using a TDC started by the beam particle trigger (done using two scintillator/photomultiplier counters in coincidence) and stopped by the first falling edge of the clock. Further, for an accepted trigger of beam particle crossing the detector, up to 16 Level 1 triggers contiguous in time were sent to the front end chip and were subsequently read-out to simulate a sequence of 16 bunch crossings; this allowed to study the timing behavior of the detector. A beam telescope consisting of 4 pairs of silicon microstrip detectors with analog readout was used to measure the transverse position of the incident beam particles. Each pair consisted of 2 planes of detectors with orthogonal strips of $50 \mu \mathrm{m}$ pitch. The resolution on the point of the trajectory extrapolated at the pixel detector varied from $4.5 \mu \mathrm{m}$ to 6.0 $\mu \mathrm{m}$, depending on the position of the detector under test with respect to the telescope.

\section{Efficiency}

Efficiency losses were studied originating either from the pixel sensor or from the analog part of the front end electronics. They are due either to a missing hit, because of a low pulse height below threshold, or to a large time walk that would cause the wrong assignment of the hit to a bunch crossing different from the expected one (from now on BX 0). To measure the efficiency, the intersection of the trajectory of a beam particle with the plane of the pixel detector was calculated; the number of expected hits was evaluated by requiring the extrapolated point to be at least $40 \mu \mathrm{m}$ from the edge of the detector (to prevent the resolution of the telescope to bias the measurement of the efficiency). When a hit was expected, 4 classes were considered:

- no hit was found (missing hits);

- a hit was found near the extrapolated point in the expected bunch crossing (good hits);

- a hit was found near the extrapo-

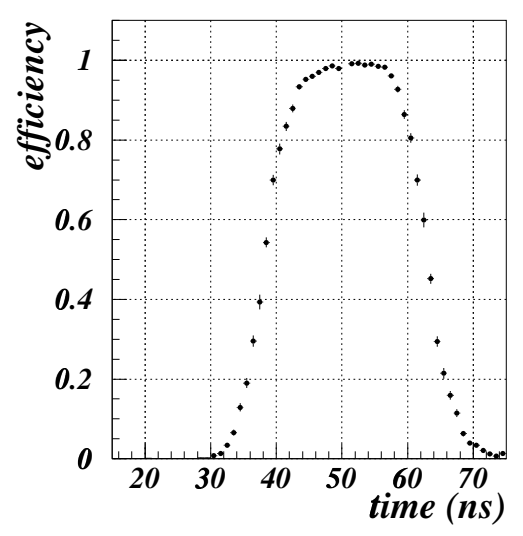

Fig. 3. Efficiency versus time for a sensor of the first Tile 2 design. 


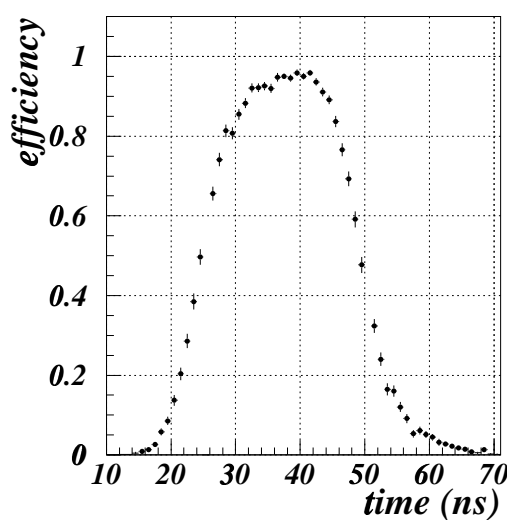

Fig. 4. Efficiency versus time for a sensor of the first Tile 2 design after irradiation.

lated point but not in the expected bunch crossing (timing losses);

- a hit was found far from the extrapolated point (tracking losses).

The good hits class was further classified depending on the number of adjacent hits. For each class the fraction with respect to the expected number of hits was calculated as a function of the beam particle phase. The efficiency as a function of the beam particle phase for $\mathrm{BX}-1$, BX 0 and $\mathrm{BX}+1$ is shown in Fig. 3 for a Tile 2 type sensor; for convenience the $3 \mathrm{BX}$ were put on the same figure with the following convention: BX -1 from 0 to $25 \mathrm{~ns}$, BX 0 from 26 to 50 ns and BX +1 from 51 to 75 ns. We observe a region of almost constant efficiency extending for about 9 ns from 48 ns to 56 ns. Similar plots for Tile 1 type sensors showed a flat region extending approximately $11 \mathrm{~ns}$. The efficiency versus time for a Tile 2 type sensor after an irradiation equivalent to $10^{15} \mathrm{n}_{\mathrm{eq}} / \mathrm{cm}^{2}$ is shown in Fig. 4; the region of constant efficiency is now reduced to approximately 5 ns. The

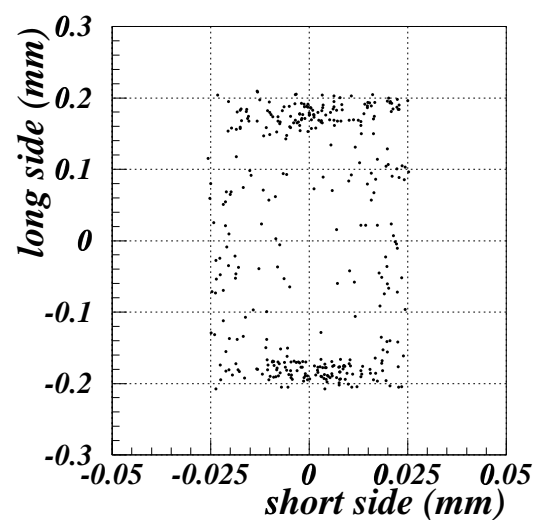

Fig. 5. Position of the missing hits for an irradiated Tile 2 sensor.

classes introduced previously were studied in the time windows of almost flat efficiency described before; the results are shown in table 1 . It was not possible to operate the Tile 1 sensor after an irradiation equivalent to $10^{15} \mathrm{n}_{\mathrm{eq}} / \mathrm{cm}^{2}$; although the Tile 1 design had efficiency performances superior to the Tile 2 design it did not survive the radiation damage foreseen for the LHC environment. For a deeper understanding we studied the position dependence of the losses; as an example, Fig. 5 shows the position of the missing hits with respect to center of the pixel cells for an irradiated sensor of the first Tile

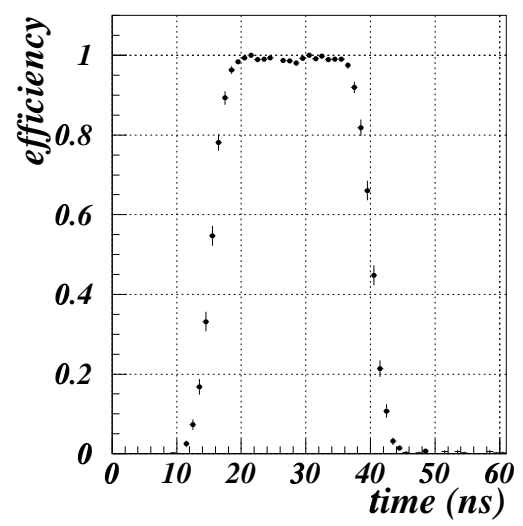

Fig. 6. Efficiency versus time for a sensor of the new Tile 2 type design. 
2 design: the losses are clearly localized at the edges of the pixel cells. Similar results were obtained studying the position of the timing losses. As will be shown in section 3 the first Tile 2 design had a charge collection inefficiency in the region of the floating atoll. A new design was realized to correct this problem removing the floating atoll and improving the bias grid geometry (Fig. 2); the new sensors were tested in a test beam in may 1999; the efficiency versus time for a new Tile 2 type sensor is shown in Fig. 6 and the detailed study of the efficiency in table 1 . An efficiency better than $99 \%$ was obtained with a flat top of 16 ns. Studies of the new design after radiation damage are in progress; however, to get an estimate of the efficiency for an irradiated Tile 2 design without the charge loss problem, a measurement of the efficiency was done requiring that the beam particles crossed the pixel in a region within $\pm 150 \mu \mathrm{m}$ and $\pm 10 \mu \mathrm{m}$ from the center of the pixel cell along the long and the short dimension respectively. A flat top of approximately 9 ns and an efficiency of $98.4 \%$ were obtained. The results are summarized in the last column of table 1 .

\section{Charge Collection}

The ability of the front end electronics to measure the pulse height with the time over threshold technique gave the possibility to measure the charge collected by the pixels and to study it as a function of the im- pact point impact of traversing particles relative to center of a pair of pixel cells. For hits not at the pixel center, the signal is shared between several pixels. The sum of the pixel pulse height (cluster pulse height) is given. During these measurements the electronics threshold was set at 2000 electrons. This threshold consequently reduced the amount of charge that could be measured in regions where it was collected by more than one pixel. The average charge

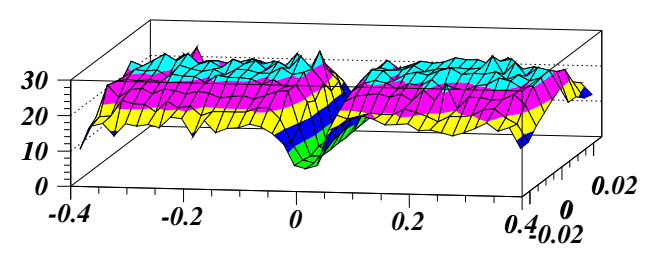

Fig. 7. Average charge collected as a function of the transverse beam position for the first Tile 2 sensor design.

collected as a function of positions $x$ and $y$ is shown in Fig. 7 where the coordinate axes are parallel to the short $(50 \mu \mathrm{m})$ and long $(400 \mu \mathrm{m})$ edges of the pixels, respectively, and are centered (in $x$ ) at the center of a single pixel and (in $y$ ) between two adjacent pixels. The average charge collected at the center of the pixel was $28 \cdot 10^{3}$ electrons; regions with reduced charge collection were ob-

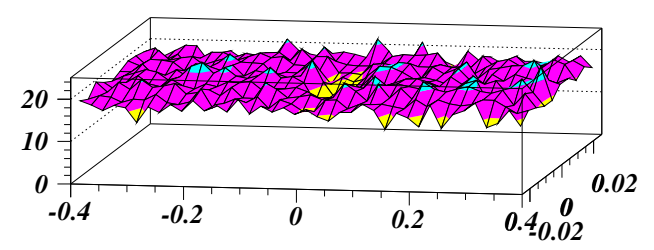

Fig. 8. Average charge collected as a function of the transverse beam position for the new Tile 2 sensor design. 
served for the Tile 2 design; these regions are located at the center of the plots in $y$ or at the edges of the pixels in both $x$ and $y$. For the Tile 2 design, charge loss was observed at all edges of the pixel. A possible explanation of this is that the $\mathrm{n}^{+}$implant ring that surrounds the main pixel implant (and which was introduced to minimize inter-pixel capacitance) did not float as it should, due to the presence of a current path not evident in the two-dimensional simulations used to optimize the design. It consequently collected signal charge of which part was then lost due to capacitive coupling to parasitic nodes (e.g. the bias grid). (A study using three-dimensional simulation is in progress to better understand this.) At the end of each Tile 2 pixel that is directly adjacent to the bias grid, two charge loss effects combine: the capacitive effect described above and a direct loss to the implanted bias dot. In this region a relatively large amount of charge loss is visible, extending approximately $\pm 50 \mu \mathrm{m}$ into the pixel region in the $y$ direction; this loss profile corresponds roughly to the geometry of the bias structure. The first charge loss effect is absent from the new Tile 2 design, which has no $\mathrm{n}^{+}$implant ring as can be seen from Fig. 8. Results for this design show only a small charge collection inefficiency at alternate gaps, corresponding to the presence of the bias grid, which includes an $\mathrm{n}^{+}$implantation line. Tile 1 sensors show only a small charge collection inefficiency on all sides of each pixel. The small gap between the individual p-stop rings acts (due to electron accumulation) like a small $\mathrm{n}^{+}$region

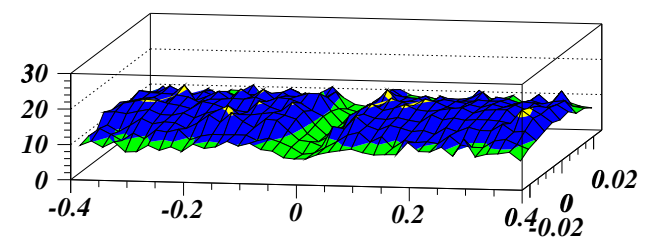

Fig. 9. Average charge collected as function of the transverse beam position for the first Tile 2 sensor design after irradiation.

[2]. Finally, in Fig. 9 the charge collection as a function of the beam particle position is shown for a Tile 2 type sensor irradiated with a fluence of $10^{15} \mathrm{n}_{\mathrm{eq}} / \mathrm{cm}^{2}$. The sensor $(280 \mu \mathrm{m}$ thick) was operated with a depletion potential of $-600 \mathrm{~V}$; as will be shown in section 5 under this bias condition the sensor was only partially depleted, the depletion depth being approximately $190 \mu \mathrm{m}$. The charge average collected at center of the pixel was $15 \cdot 10^{3}$ electrons and the charge losses at the edge of the pixel cells were similar to the not irradiated Tile 2 sensor.

\section{Resolution}

The spatial resolution was measured by computing the residuals between the hit position predicted by the silicon microstrip telescope and that from the pixel detector. Single pixel and double pixel clusters were studied separately; double pixel clusters were studied using two different algorithms: a binary algorithm, in which the measured cluster position was taken at the center of the 2 hit pixels and an analog algorithm in which the binary position was cor- 


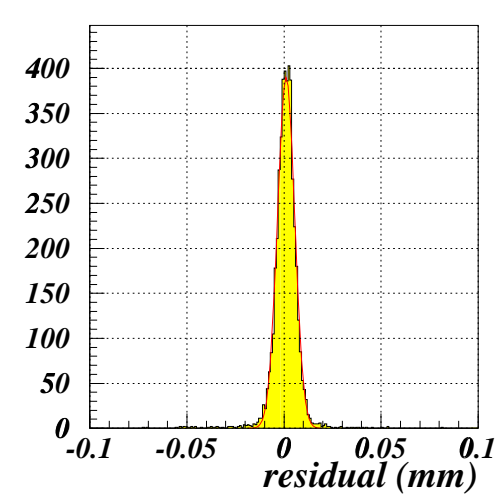

Fig. 10. Distribution of the residuals for the 2 pixels clusters using the analog algorithm.

rected using the measured charge collected by the 2 hit pixels. The charge sharing between 2 pixels was studied separately: for the $280 \mu \mathrm{m}$ thick sensor of Tile 2 type, with a threshold of 3000 electrons, the region of charge sharing was limited to approximately $\pm 6 \mu \mathrm{m}$ around the center of the 2 pixel cells; this region was reduced to approximately \pm 4 $\mu \mathrm{m}$ for the $200 \mu \mathrm{m}$ thick sensors. The distribution of the residuals of a $200 \mu \mathrm{m}$ thick sensor for the analog and binary algorithm is shown in Fig. 10 and Fig. 11 respectively. For these measurements, the error on the predicted hit position was $4.2 \mu \mathrm{m}$,

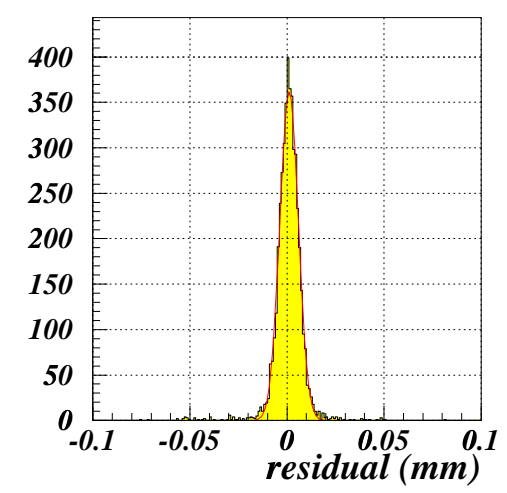

Fig. 11. Distribution of the residuals for the 2 pixels clusters using the binary algorithm. calculated taking into account the resolution of the silicon microstrip planes and the multiple scattering due to material of the experimental setup. The width of the residuals was calculated fitting the distributions with a Gaussian function and gave $4.4 \mu \mathrm{m}$ and $4.8 \mu \mathrm{m}$ for the analog and digital algorithm respectively. The single pixel cluster residual distribution is shown in Fig. 12. The

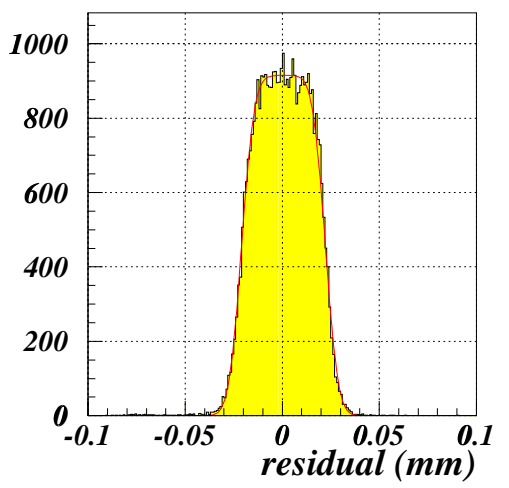

Fig. 12. Distribution of the residuals for the 1 pixel clusters.

distribution was parametrized with a uniform distribution of width $\mathrm{L}$, as expected for the single pixel cluster residuals, convoluted with a Gaussian distribution that took into account the resolution of the silicon strip telescope. The result of the fit gave $\sigma=5.2 \pm .1 \mu \mathrm{m}$ and $\mathrm{L}=43.2$ $\pm 0.2 \mu \mathrm{m}$; the fitted width $\mathrm{L}$ is in agreement with the measured width of $\pm 4 \mu \mathrm{m}$ of the region in which charge sharing occurs. The single pixel and double pixel cluster residual were put together and the resulting distribution is shown in Fig. 13. The rms of the distribution was 13.4 $\mu \mathrm{m}$; subtracting the resolution of the silicon telescope a value of $12.7 \mu \mathrm{m}$ was obtained that is a measurement of the resolution of the sensor for 


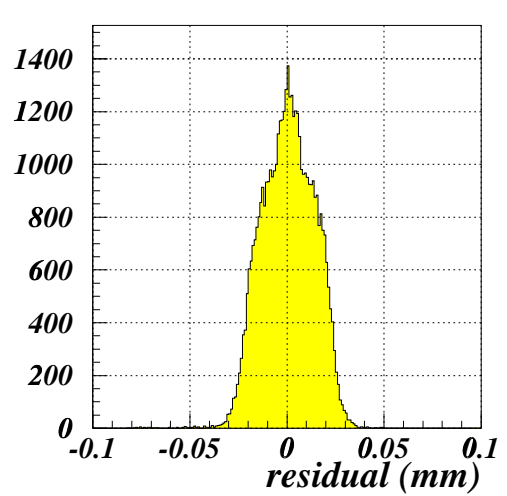

Fig. 13. Distribution of the residuals for the 1 pixel clusters and 2 pixels clusters using the binary algorithm.

normal incidence of the beam particles. The relative weight of the single pixel and double pixels clusters can be seen from table 1 were the fraction of double hits is given. Finally, the dependence of the resolution on the angle of the incident particle was studied. For each angle of incidence of the track, the rms of the residuals distribution (single and double pixel cluster) was measured using the binary and the analog algorithm. The results are shown in Fig. 14 where also the rms of the residuals measured with a sensor irradiated at a fluence of $10^{15} \mathrm{n}_{\mathrm{eq}} / \mathrm{cm}^{2}$ is also plotted. The data were not corrected for the silicon strip telescope resolution.

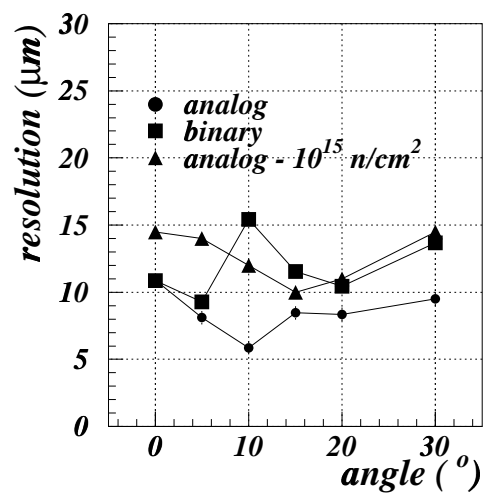

Fig. 14. Width (rms) of the residuals versus the track incidence angle.
It can be noticed that at an angle of $10^{0}$ the analog algorithm allows to obtain a resolution better than 6 $\mu \mathrm{m}$. This improvement was due to the increased cluster size and thus increased charge sharing between neighboring pixels. The irradiated detector resolution never reaches values below $10 \mu \mathrm{m}$ because of the small fraction of double pixel cluster (7.6 $\%)$. However it should be noticed that this is an old Tile 2 design, suffering the charge collection problems discussed in section 3 , and an improvement is expected with the new Tile 2 design.

\section{$5 \quad$ Depleted depth}

The choice of the $\mathrm{n}^{+} / \mathrm{n}$ technology has the consequence that during the operation in the LHC environment the silicon bulk material will undergo a type inversion becoming of $p$ type. The variation of the effective doping and the increase of the leakage current will eventually prevent the fully depleted operation of the detector. The depletion depth of two Tile 2 sensors, irradiated with a fluence of $0.5 \times 10^{15}$ and $10^{15} \mathrm{n}_{\mathrm{eq}} / \mathrm{cm}^{2}$ respectively, was measured. To measure the depletion depth data were taken with the sensor at an angle of $30^{0}$ with respect to the direction of the beam along the short direction of the pixel cells. The method is illustrated in Fig. 15: a beam particle crossing the detector produced a cluster consisting of the pixel cells which collected the charge of the subtended segment of the track; for 


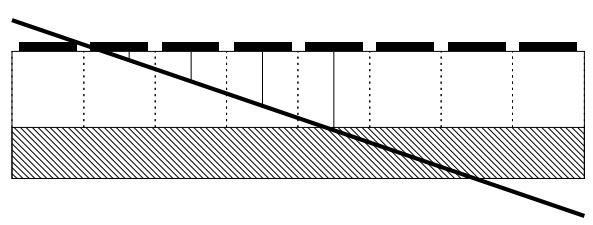

Fig. 15. Illustration of the measurement of the depletion depth.

each segment the depth of the center of the segment was calculated. The distribution of the depths is shown in Fig. 16; from the figure the maximum depth at which the charge was still collected can be measured: to fit the rightmost part of the distribution of the depth $d$ the function $f(d)=a\left(1-\tanh b\left(d-d_{\max }\right)\right)$ was used where $a$ was a normalization factor, $b$ a width parameter, and $d_{\text {max }}$ the maximum depth. From the fit we obtained $d_{\max }=190 \pm 14$ $\mu \mathrm{m}$ for the sensor irradiated with a dose equivalent to $10^{15} \mathrm{n}_{\mathrm{eq}} / \mathrm{cm}^{2}$ and $d_{\max }=265 \pm 11 \mu \mathrm{m}$ for the sensor irradiated with $0.5 \times 10^{15} \mathrm{n}_{\mathrm{eq}} / \mathrm{cm}^{2}$. Both detectors were $280 \mu \mathrm{m}$ thick and were biased at $-600 \mathrm{~V}$. It can be noticed that the Tile 2 sensors are almost fully depleted after a dose of $0.5 \times 10^{15} \mathrm{n}_{\mathrm{eq}} / \mathrm{cm}^{2}$ when biased at $-600 \mathrm{~V}$.

\section{The Lorentz angle}

The ATLAS pixel detector will operate in a magnetic field of 2 Tesla; in the barrel part of the detector, the magnetic field $\vec{B}$ and the electric drift field $\vec{E}$ inside the silicon pixel detector will be orthogonal and the drift velocity will acquire a compo-

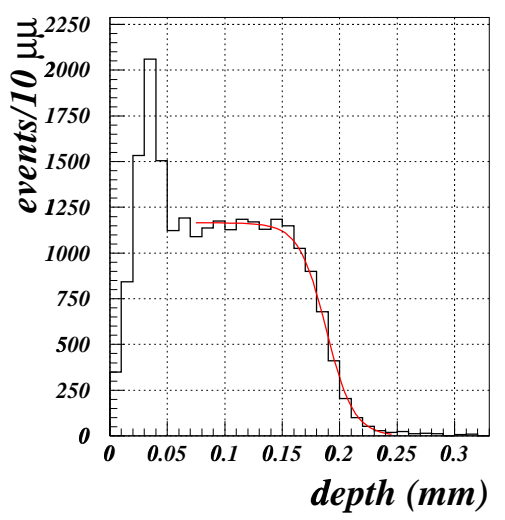

Fig. 16. Distribution of the depth of the charge segments for an irradiated sensor.

nent along the direction of $\vec{E} \times \vec{B}$; the charge carrier will then drift at an angle $\theta_{L}$ with respect to direction of the electric field, $\theta_{L}$ being the Lorentz angle[7]. Depending on the angle of the incident particle, the charge collected will spread over several pixel cells, the spread being minimum for an angle of the incident particle equal to $\theta_{L}$. The Lorentz angle for irradiated and not irradiated detector of the Tile 2 design was measured. The method used is well known [8]: the mean cluster size was measured as a function of the angle of the incident beam particles with respect to the normal to the detector. For each angle 2 measurements were performed with magnetic field off, $\vec{B}=0$, and magnetic field on $\vec{B}=1.4$ Tesla. The mean cluster size as function of the angle for a not irradiated detector operated at a bias voltage of $-150 \mathrm{~V}$ is shown in Fig. 17; a fit to data is also shown. The fitting function was constructed using a simple model in which the ionization charge produced along the particle track was divided in small segments and each segment was propagated 


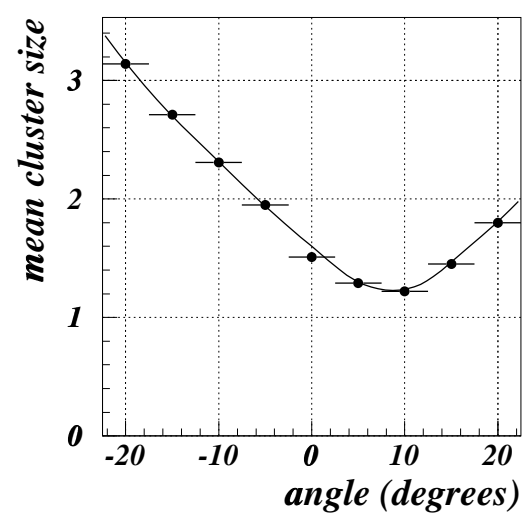

Fig. 17. Mean cluster size as function of the beam angle for a detector before irradiation.

towards the pixel cells taking into account the Lorentz angle and the diffusion. Once all the charge was collected by the pixel cells, a threshold was applied and the cluster size was evaluated. This procedure was repeated for different positions of the incident beam particle with to respect to the pixel cells. The mean cluster size was then evaluated. The model described did not take into account yet the Landau fluctuations. The fit was performed both on the magnetic field on and magnetic field off data. With magnetic field off the Lorentz angle should be zero and a result different from zero was taken as systematic error for the fit with magnetic field on. The results obtained were $\theta_{L}=9.1^{0} \pm 0.1^{0} \pm 0.6^{0}$ for a not irradiated sensor operated at $-150 \mathrm{~V}, \theta_{L}=3.0^{0} \pm 0.5^{0} \pm 0.2^{0}$ for a sensor irradiated with $0.5 \times 10^{15}$ $\mathrm{n}_{\mathrm{eq}} / \mathrm{cm}^{2}$ and operated at $-600 \mathrm{~V}$ and $\theta_{L}=3.2^{0} \pm 1.2^{0} \pm 0.5^{0}$ for a sensor irradiated with $10^{15} \mathrm{n}_{\text {eq }} / \mathrm{cm}^{2}$ and operated at $-600 \mathrm{~V}$, where the first error is statistical and the second one systematic.

\section{Conclusions}

A big effort was undertaken within the ATLAS Collaboration to finalize the pixel detector project. Different sensor designs were studied in a test beam and detailed studies of the efficiency were presented and discussed. The $p$-spray insulation technique was proven to be radiation resistant and efficiencies exceeding $99 \%$ with time plateau of 16 ns were measured. The first Tile 2 design had a charge collection inefficiency due to a floating atoll that did not float as it should, due to the presence of a current path not evident in the two-dimensional simulations used to optimize the design; a new Tile 2 design was developed that removed the charge collection inefficiency. The charge sharing between adjacent pixel cells was studied and found to be confined within $\pm 4 \mu \mathrm{m}$ and $\pm 6 \mu \mathrm{m}$ around the center of the 2 pixels for $200 \mu \mathrm{m}$ and 280 $\mu \mathrm{m}$ thick sensors respectively. Binary and analog algorithms for the coordinate measurement were studied and the resolution as a function of the incident angle of the track measured: a resolution better than $6 \mu \mathrm{m}$ was measured at an angle of incidence of $10^{\circ}$. The depleted depth of a $280 \mu \mathrm{m}$ thick detector irradiated with a fluence of $10^{15} \mathrm{n}_{\mathrm{eq}} / \mathrm{cm}^{2}$ was measured to be 190 $\pm 14 \mu \mathrm{m}$ with a bias voltage of -600 $\mathrm{V}$. The Lorentz angle in a field of 1.4 Tesla varied from $\theta_{L}=9.1^{0} \pm 0.1^{0} \pm$ $0.6^{0}$ for a not irradiated sensor operated at $-150 \mathrm{~V}$ to $\theta_{L}=3.2^{0} \pm 1.2^{0} \pm$ $0.5^{0}$ for a sensor irradiated with $10^{15}$ $\mathrm{n}_{\text {eq }} / \mathrm{cm}^{2}$ and operated at $-600 \mathrm{~V}$ and at a temperature of $-10^{0} \mathrm{C}$. 


\section{Acknowledgements}

The work reported here represents the joint efforts of many individuals in the ATLAS pixel Collaboration. I would like to thank all of them.

\section{References}

11 The ATLAS Pixel Collaboration, "ATLAS Pixel Detector Technical Design Report", LHCC 98-13, CERN 1998.

[2] The ATLAS Pixel Collaboration, "The ATLAS Silicon Pixel Sensors", Nucl. Instr. and Meth., submitted for publication.

[3] G. Batignani et al., Nucl. Instr. and Meth. A 277 (1989) 147.

[4] R.H. Richter et al., Nucl. Instr. and Meth. A 377 (1996) 412.

[5] R. Wunstorf, IEEE Trans. on Nucl. Sci. 44(3) (1997) 806.

[6] H. Feick, "Radiation Tolerance of Silicon Particle Detectors for H.E. Physics Experiments", Ph. D. thesis University Hamburg and DESY internal report F35D 9708 (1997).

[7] S.R. Amendolia et al., Nucl. Instr. and Meth. A 235 (1985) 296.

[8] N. Bingefors et al., Nucl. Instr. and Meth. A 328 (1993) 447. 
Table 1

Efficiency measurement summary.

\begin{tabular}{lrrcrc}
\hline & \multicolumn{2}{c}{ Old Design } & New Design & \multicolumn{2}{c}{ Old Design } \\
& \multicolumn{2}{c}{$280 \mu \mathrm{m}$} & $200 \mu \mathrm{m}$ & \multicolumn{2}{c}{$10^{15} \mathrm{n} / \mathrm{cm}^{2}$} \\
& \multicolumn{1}{c}{$T 2$} & \multicolumn{1}{c}{$T 1$} & \multicolumn{1}{c}{$T 2$} & \multicolumn{1}{c}{$T 2$} & $T 2^{*}$ \\
\hline 1 hit & 82.0 & 72.0 & 81.8 & 86.3 & 94.2 \\
2 hits & 14.6 & 25.2 & 15.6 & 7.6 & 3.1 \\
$\geq 3$ hits & 2.2 & 2.4 & 1.7 & 1.4 & 1.1 \\
\hline efficiency & 98.8 & 99.6 & 99.1 & 95.3 & 98.4 \\
\hline losses & 1.2 & 0.4 & 0.9 & 4.7 & 1.6 \\
\hline 0 hits & 0.4 & 0.1 & 0.4 & 2.2 & 0.4 \\
tracking & 0.2 & 0.2 & 0.1 & 0.1 & 0.0 \\
timing & 0.6 & 0.1 & 0.4 & 2.4 & 1.2 \\
\hline
\end{tabular}

${ }^{*}$ with position cut, see text 